\title{
PROGRESIÓN DE LAS IDEAS DEL PROFESORADO EN FORMACIÓN SOBRE LAS PRÁCTICAS DE CAMPO EN UNA SECUENCIA DE APRENDIZAJE
}

\author{
Elias Francisco Amortegui Cedeno ${ }^{1}$ \\ Valentin Gavidia Catalan ${ }^{2}$ \\ Olga Mayoral García-Berlanga ${ }^{3}$
}

\begin{abstract}
RESUMEN
Las Prácticas de Campo adquieren un valor especial en la enseñanza y aprendizaje de la Biología pues permiten al alumnado abordar su objeto de estudio, "lo vivo», lo más cerca posible a sus condiciones naturales, con una perspectiva sistémica y holística que les permite comprender la relaciones que conforman el fenómeno viviente en conjunto con su ambiente, así como potenciar el aprendizaje del conocimiento biológico, destrezas y habilidades del trabajo de campo y sobre todo favorece la adquisición de actitudes sobre el cuidado de los ecosistemas; pese a esto, son escasas las investigaciones sobre la incidencia de las Prácticas de Campo en la formación inicial del profesorado y más aún en la construcción de su saber profesional docente. Realizamos aquí una investigación con 27 docentes en formación del Programa de Licenciatura en Ciencias Naturales y Educación de la Universidad Surcolombiana (Neiva-Colombia). El estudio es de tipo mixto, prospectivo y longitudinal, empleando análisis de contenido y los Software SPSS y Atlas.Ti. Para el caso de este artículo, se presenta el análisis de la aplicación de un pre y postest; mostramos respuestas de los futuros docentes y el valor estadístico de la t-student al comparar ambos momentos. El estudio pone de manifiesto la contribución de un seminario formativo en la progresión de las concepciones del profesorado en formación sobre la naturaleza de las Prácticas de Campo, sus finalidades de aprendizaje, su planificación y el aporte a la formación docente.
\end{abstract}

Palabras-clave: Prácticas de campo. Formación inicial. Enseñanza de la biología.

\section{PROGRESSION OF THE IDEAS OF TEACHERS IN TRAINING ON FIELD PRACTICES IN A LEARNING SEQUENCE}

\section{ABSTRACT}

The Field Practices acquire a special value in the teaching and learning of Biology because they allow the students to approach their object of study, "the living", as close as possible to their natural conditions, with a systemic and holistic perspective that allows them to understand the relationships that make up the living phenomenon in conjunction with its environment, as well as enhancing the learning of biological knowledge, skills and abilities of field work and above all favors the acquisition of attitudes about the care of ecosystems; Despite this, there is little research on the incidence of Field Practices in the initial teacher training and even more in the construction of their teaching professional knowledge. We carried out an investigation with 27 teachers in formation of the Degree Program in Natural Sciences and Education of the Surcolombiana University (Neiva-Colombia). The study is mixed, prospective and longitudinal, using content analysis and the SPSS and Atlas.Ti Softwares. For the case of this article, the analysis of the application of a pre- and post-test is presented; we show the answers of the future teachers and the statistical value of the t-student when comparing both moments. The study highlights the contribution of a training seminar in the progression of teachers' conceptions in training on the nature of field practices, their learning purposes, their planning and the contribution to teacher training.

Keywords: Field practice. Initial training. Teaching biology.

Recebido em: $16 / 1 / 2020$

Aceito em: $24 / 3 / 2020$

\footnotetext{
${ }^{1}$ Docente de planta tiempo completo. Programa de Licenciatura en Ciencias Naturales y Educación Ambiental. Universidad Surcolombiana, Colombia. http://orcid.org/0000-0001-9179-1503. amortegui@usco.edu.co

2 Departamento de Didáctica de la Ciencias Experimentales y Sociales. Universitat de València, España. http://orcid.org/0000-0001-9153147X.gavidia@uv.es

3 Departamento de Didáctica de la Ciencias Experimentales y Sociales. Jardí Botànic de la Universitat de València, España. https://orcid. org/0000-0003-2340-4676. mayoral@uv.es
} 
En el marco de la enseñanza y aprendizaje de la Biología, y específicamente de la Ecología, son múltiples las dificultades planteadas por diferentes autores (BANET, 2000; JIMÉNEZ, 2003; ARTOLA; MAYORAL; BENARROCH, 2016): la noción de ecosistema; la percepción lineal de las relaciones (cadenas, no redes); la concepción estática de su funcionamiento; la deficiente interpretación de redes alimentarias y la escasa sensibilización acerca del impacto del ser humano en la dinámica existente en su entorno. Sin embargo, un adecuado planteamiento de las Prácticas de Campo ofrece oportunidades educativas de incuestionable valor que pueden ayudar a superar diversas de estas dificultades, con el valor añadido de involucrar las dimensiones afectiva, cognitiva y procedimental.

En ocasiones, la mayor relación que tiene el alumnado con los organismos vivos tiene lugar en zoológicos o acuarios (MCLAUGHLIN; JOHNSON, 2006) por lo que consideramos que las Prácticas de Campo son fundamentales para superar estas deficiencias, ampliar los horizontes del alumnado y facilitar al docente una transposición didáctica más efectiva (LAVIE ALON; TAL, 2015). Ofrecen oportunidades educativas de alto valor relacionadas con aspectos procedimentales y actitudinales, como son la apreciación del significado de naturaleza, la valoración y conservación y disfrute sostenible de los recursos naturales, entre otros (MAGNTORN; HELLDÉN, 2005, 2007; HAMILTON-EKEKE, 2007). Es importante promover una Biología escolar que tenga en cuenta el respeto y la valoración de los seres vivos que se estudian (GRILLI-SILVA, 2018), en nuestro caso, en su ambiente natural. Como afirman Brody (2005), Morag y Tal (2012) y Morag, Tal y Rotem-Keren (2013), las Prácticas de Campo en ambientes naturales se diferencian de las visitas a diferentes museos, zoológicos, jardines botánicos o centros de ciencia, pues permiten la experiencia directa con los fenómenos reales de la naturaleza; más que cualquier otra actividad extra-escolar.

Una de las mayores dificultades que afronta la enseñanza de la biología en entornos naturales consiste en que los docentes cuentan con una preparación insuficiente sobre cómo enseñar en la propia naturaleza (DEL TORO; MORCILLO, 2011; ATESKAN; LANE, 2016). Generalmente el profesorado, en su formación inicial, ha participado como alumnado en las Salidas de Campo, sin tener experiencias en cómo deben planificar y enseñar fuera del aula (TAL; MORAG, 2009; AMÓRTEGUI; CORREA, 2012). Amórtegui, Mayoral y Gavidia (2017) han puesto de manifiesto el reducido número de investigaciones sobre las Prácticas de Campo como estrategia de enseñanza. Algunas de éstas se han venido centrando en abordar las concepciones del profesorado, mientras que su aporte a la construcción del CPP sigue siendo un campo de investigación vacío. Por ello, el objetivo del presente trabajo es caracterizar la contribución que realiza el diseño, desarrollo y evaluación de un Seminario sobre las Prácticas de Campo a la construcción del Conocimiento Profesional del Profesorado de los futuros docentes de Biología, y en concreto, estudiar la progresión de sus concepciones. 


\section{ASPECTOS METODOLÓGICOS}

\section{Contexto y Muestra}

Ubicamos nuestro estudio en el contexto colombiano. En concreto, nos centramos en la formación del profesorado de Ciencias Naturales y Educación Ambiental en el Departamento del Huila (Sur del país), de la Universidad Surcolombiana (carácter público). La muestra de estudio está formada por el $100 \%$ de los estudiantes que cursaron Didáctica I (sexto semestre) durante el segundo semestre del 2016 (julio a diciembre). El grupo consiste en 27 estudiantes ( 21 mujeres y 6 hombres) cuyas edades oscilan entre los 19 y 23 años. Al inicio del curso se les manifestó la intencionalidad del estudio y la totalidad de los asistentes del mismo manifestaron su participación voluntaria a través de un consentimiento informado. Al indicar los 5 grupos de trabajo arriba, se han puesto los cursos a los que se dirigían las salidas de campo. Creo que es esencial añadir una frase en la que se diga las edades de los niños con los que se ha trabajado en última instancia.

\section{Desarrollo del Seminario}

Se ha llevado a cabo el seminario Las Prácticas de Campo en la enseñanza de la biología y la formación docente (Anexo 1 ) en el que se ha tenido en cuenta lo que el futuro profesorado necesitará para el desarrollo de este tipo de actividades, esto es: su diseño y preparación, ejecución y evaluación. Ateniendo a la necesidad de caracterizar las aportaciones del seminario en la formación inicial del profesorado, decidimos realizar nuestra investigación con una perspectiva mixta, que contempla tanto los aspectos cualitativos como los cuantitativos, lo que permite destacar con mayor efectividad sus contribuciones a la formación del profesorado (VAN DRIEL; BERRY; MEIRINK, 2014). Nuestro estudio ha sido de tipo prospectivo longitudinal, que pone de manifiesto la progresión del propio aprendizaje profesional del profesorado (RIVERO et al., 2017).

El desarrollo del seminario se llevó a cabo de forma que el profesorado en formación inicial se organizó en cinco grupos que abordaron temáticas biológicas particulares aplicadas a cursos (niveles educativos) específicos de educación básica secundaria con estudiantes entre los 10 y 15 años de edad de instituciones educativas públicas de la ciudad de Neiva (Colombia) durante sus períodos de prácticas. Estos grupos fueron: G1) ecosistemas y redes tróficas (alumnado de 8 ㅇ grado), G2) insectos y sus ambientes ( 8 은 grado), G3) plantas (7ㅇ grado), G4) artrópodos (6 grado) y G5) insectos (6ㅇg grado). Todos los grupos tuvieron la siguiente organización: entre una y dos sesiones previas de preparación en el aula con el alumnado (2 horas); una mañana para su aplicación (3-4 horas); y una o dos sesiones para la conclusión de la salida en el aula ( 2 horas). En total la secuencia implicó en torno a 10 horas. Las Prácticas de Campo se llevaron a cabo: en dos casos, en el bosque de la propia institución educativa (G3, G5); en otros dos casos en un bosque que contenía una quebrada cerca de la escuela $(\mathrm{G} 1, \mathrm{G} 2)$; un grupo trabajó 
en un humedal a las afueras de la ciudad al que accedieron en autobús (G4). La planificación del taller con objetivos, contenidos y actividades de desarrollo se encuentra en el Anexo 1.

\section{Instrumentos de Recolección y Análisis de Datos}

Entre septiembre y diciembre del año 2016 se llevó a cabo durante 9 sesiones de clase un seguimiento mediante observación participante. Cada sesión tuvo una duración de 2 horas, en las que realizamos el seguimiento del trabajo de los futuros docentes en las respectivas instituciones educativas donde desarrollaron su Práctica de Campo. Todas las sesiones fueron grabadas en video y luego transcritas y sistematizadas para su análisis posterior. Para ello se ha organizado la información atendiendo al análisis del contenido (PÉREZ, 1994; ÁLVAREZ; JURGENSON, 2003) a través del Software Atlas.Ti 7.0 y, para el caso del cuestionario, se ha aplicado una $t$-student, siguiendo las orientaciones de Cohen, Manion y Morrison (2011). Las herramientas utilizadas para la obtención de datos acerca de los resultados del seminario han sido: a) un cuestionario (aplicado como pre y postest), b) la observación participante (mediante el análisis de la video-grabación del desarrollo de la intervención didáctica) y c) el seguimiento al desarrollo del seminario a través de las entregas consecutivas por parte del alumnado. En este artículo nos centraremos en los resultados de la aplicación del primer instrumento, es decir, el cuestionario. El cuestionario consta de dos secciones: en la primera, de seis preguntas, se abordan aspectos generales sobre las Salidas de Campo; en la segunda se plantea un escenario hipotético sobre el que deben contestar a seis situaciones diferentes en las que pueden encontrarse como docentes en su región. Dicho cuestionario es una modificación de uno anterior validado por expertos en Didáctica de la Biología de Colombia (Universidad Pedagógica Nacional) y de España (Universidad de Extremadura) (AMÓRTEGUI, 2011). Para el análisis de los resultados se estableció un sistema de categorías que se refieren a los aspectos sustanciales sobre las Prácticas de Campo. En el presente estudio hemos concretando la propuesta de Puentes (2008) y Amórtegui y Correa (2012), atendiendo a la revisión bibliográfica realizada y se han adicionado las categorías Naturaleza del Trabajo de Campo, Planificación y Dificultades. En la Tabla 1 se muestran las preguntas del cuestionario, las categorías y subcategorías, resaltando con sombreado aquellas que han sido analizadas en el presente trabajo, ante la imposibilidad, por límites de espacio, de presentar el análisis de todas ellas.

Tabla 1 - Categorías para la evaluación de las Prácticas de Campo en la enseñanza de la biología y la formación docente (en sombreado, las categorías que se analizan)

\begin{tabular}{|l|l|}
\hline \multicolumn{1}{|c|}{ PREGUNTA } & \multicolumn{1}{|c|}{$\begin{array}{c}\text { CATEGORÍA A } \\
\text { INDAGAR }\end{array}$} \\
\hline 1.1 Para ti, ¿qué es una Práctica de Campo? & $\begin{array}{l}\text { NATURALEZA DEL } \\
\text { TRABAJO DE CAMPO }\end{array}$ \\
\hline $\begin{array}{l}1.2 \text { ¿Qué relaciones encuentras entre las Prácticas de Campo y los temas } \\
\text { trabajados en las clases de Ciencias Naturales? }\end{array}$ & $\begin{array}{l}\text { RELACIÓN TEORÍA- } \\
\text { PRÁCTICA }\end{array}$ \\
\hline $\begin{array}{l}\text { 1.3 ¿Qué consideras que aprenden los alumnos cuando realizan una Práctica } \\
\text { de Campo? }\end{array}$ & $\begin{array}{l}\text { FINALIDADES DE } \\
\text { APRENDIZAJE }\end{array}$ \\
\hline $\begin{array}{l}1.4 \text { ¿Crees que una Práctica de Campo para la enseñanza de la Biología, difiere } \\
\text { en sus características de una Salida de Campo para la enseñanza de otras } \\
\text { ciencias (por ejemplo: enseñanza de la Geología, enseñanza de la Geografía)? }\end{array}$ & $\begin{array}{l}\text { ASPECTOS } \\
\text { EPISTEMOLÓGICOS }\end{array}$ \\
\hline
\end{tabular}




\begin{tabular}{|c|c|}
\hline $\begin{array}{l}5 \text { ¿Qué aportan las Prácticas de Campo a tu formación como docente de } \\
\text { encias Naturales? }\end{array}$ & $\begin{array}{l}\text { APORTE FORMACIÓN } \\
\text { DOCENTE }\end{array}$ \\
\hline $\begin{array}{l}.6 \text { ¿Qué imagen de Biología desarrollan los alumnos cuando realizan una } \\
\text { ráctica de Campo? }\end{array}$ & $\begin{array}{l}\text { ASPECTOS } \\
\text { EPISTEMOLÓGICOS }\end{array}$ \\
\hline \multicolumn{2}{|c|}{$\begin{array}{l}\text { Eres un profesor titular de una Institución Educativa Oficial de la ciudad de Neiva; particularmente para } \\
\text { el grado noveno te encuentras enseñando Biología y tienes en mente realizar una Salida de Campo. A } \\
\text { respecto: }\end{array}$} \\
\hline 2.1 ¿Qué tendrías en cuenta para realizar la Práctica de Campo? & PLANIFICACIÓN \\
\hline $\begin{array}{l}2.2 \text { ¿Realizarías la Salida de Campo antes, durante o después de desarrollar los } \\
\text { temas en clase? }\end{array}$ & $\begin{array}{l}\text { MOMENTO DE } \\
\text { REALIZACIÓN }\end{array}$ \\
\hline $\begin{array}{l}2.3 \text { ¿Cuáles serían los roles del docente y los alumnos durante dicha Salida de } \\
\text { Campo? }\end{array}$ & $\begin{array}{l}\text { ROL DOCENTE- } \\
\text { ALUMNO }\end{array}$ \\
\hline 2.4 ¿Qué dificultades podrías tener para llevar a cabo la Práctica de Campo? & DIFICULTADES \\
\hline $\begin{array}{l}2.5 \text { ¿En qué consistiría la evaluación del aprendizaje de tus alumnos en dicha } \\
\text { Práctica de Campo? }\end{array}$ & $\begin{array}{l}\text { EVALUACIÓN DEL } \\
\text { APRENDIZAJE }\end{array}$ \\
\hline $\begin{array}{l}2.6 \text { ¿Qué aportaría la realización de esta Práctica de Campo a tu desarrollo } \\
\text { profesional? }\end{array}$ & $\begin{array}{l}\text { APORTE FORMACIÓN } \\
\text { DOCENTE }\end{array}$ \\
\hline
\end{tabular}

Fuente: Elaboración de los autores.

\section{RESULTADOS Y DISCUSIÓN}

Los resultados obtenidos tras el análisis de los cuestionarios previo y posterior permiten analizar la evolución del enfoque del futuro profesorado respecto a las Prácticas de Campo. Dado el volumen de resultados de nuestra investigación, nos centraremos en presentar: las ideas previas de los estudiantes al comienzo del proceso formativo (pre test) y la progresión de sus concepciones luego del desarrollo del mismo (pos test). Esta comparación se llevó a cabo a través de una prueba $t$ student para medias con un valor de significatividad de $0,99 \%$ y con un valor de confianza 0,95\%. En cada caso presentamos, después de la pregunta entre corchetes, algunas de las respuestas textuales mayoritarias del futuro profesorado y realizamos una discusión desde el marco de la Didáctica de las Ciencias.

\section{Análisis de los Resultados Obtenidos en los Cuestionarios Previos y Posteriores al Seminario}

\section{Categoría: Naturaleza del Trabajo de Campo}

[¿Qué es una Práctica de Campo?]

Resultados Pretest:

"Práctica de campo es dirigirse a un lugar fuera del aula de clase y de la misma universidad, ya sea a otro municipio o ciudad..."

15 estudiantes $(55,5 \%)$ se circunscriben a una postura en la que se refieren exclusivamente al hecho de salir de la escuela. En algunos casos se hace referencia a espacios de educación formal (otras universidades) y en otros a no formal (centros tecnológicos, estudios científicos, entre otros). Se hace escasa alusión a un lugar natural como un ecosistema, bosque, reserva, etc.

Resultados Postest: 
"Es una metodología didáctica que permite tener un acercamiento más profundo sobre el tema que se quiere abordar..."

Veinte y tres de los futuros docentes $(85,2 \%)$ explicitan que la Práctica de Campo es una estrategia de enseñanza que podrán desarrollar con su alumnado en su futuro quehacer como profesores. Realizan precisiones como que permitirá a su alumnado trabajar en la "realidad» bajo el "contacto directo» y les facilitará estudiar el fenómeno viviente lo más cercano a sus condiciones naturales.

En el primer test los futuros docentes no hacen referencia a elementos de tipo didáctico sobre esta Estrategia de enseñanza, tales como sus finalidades de aprendizaje, sus aportaciones, los roles de trabajo o su planificación. Se centran en una perspectiva más como aprendices que como futuros profesores y, por tanto, no consideran de manera explícita que sea una actividad de Desarrollo profesional. Este tipo de concepciones pueden reafirmar la dificultad planteada por Morag y Tal (2012) y Dourado y Leite (2013), acerca de la diversidad de acepciones que la literatura reporta sobre el término de Práctica de Campo, entre ellas field activities, educational field activities, field trips, study visits, out of school, excursión, educación experiencial, interpretación ambiental. Glackin (2016) plantea que el profesorado que se identifica con enfoques más tradicionales de enseñanza suele considerarlas como una actividad divertida para el alumnado. La comparación entre el pre y post test nos arroja un $p$-valor $\leq 0.001$, mostrando una alta significatividad de los datos entre el antes y después y, por tanto, una gran progresión en sus concepciones. A diferencia del inicio del proceso formativo, aquí el abordaje que realiza el profesorado es más como una estrategia de enseñanza fundamental en el aprendizaje de la Biología, con gran efectividad en la adquisición de conceptos, procedimientos y actitudes que permiten al alumnado comprender desde una perspectiva sistémica, el fenómeno de lo viviente.

\section{Categoría: Finalidades de aprendizaje}

[¿Qué consideras que aprenden los alumnos cuando realizan una Práctica de Campo?]

Resultados Pretest:

"Los estudiantes aprenden en una práctica de campo el funcionamiento y características, puede ser de diferentes organismos o procesos del tema que se esté trabajando. ..."

Para 21 (77,7\%) futuros docentes las Prácticas de Campo se realizan con el fin de que el alumnado corrobore los conceptos que el profesor previamente ha tratado en clase; especificando que aprenden sobre objetos de estudio concretos como plantas, animales, ambientes naturales, factores bióticos, abióticos y la naturaleza en general.

Resultados Postest:

"Es muy bonito que el estudiante interactúe con el medio ambiente y conozca su entorno, respetando y conservando, así este tomará una nueva perspectiva a la temática que se plantea...". 
Las respuestas al primer test poseen una perspectiva donde predominan los contenidos Conceptuales. Las Prácticas de Campo no suelen estar enmarcadas dentro de un problema, ni en un contexto específico, tan solo muestran un fenómeno biológico, en el que no existe espacio a su discusión o construcción. No se hace evidente ninguna intervención sobre el objeto de estudio, por tanto, basta con la mera observación del fenómeno de estudio (DEL CARMEN; PEDRINACI, 1997; DOURADO; LEITE, 2013).

Respuestas al postest: Aquí la subcategoría Actitudinal fue en la que encontramos la más alta significatividad, ( $p$-valor $\leq 0.001$ ) entre sus respuestas de antes y después del desarrollo del seminario, y observamos la mayor modificación en el número de estudiantes que concuerdan con esta subcategoría, pasando de $12(44,4 \%)$ a $25(92,6 \%)$ futuros docentes, quienes afirman que en el campo, los alumnos aprenden sobre la responsabilidad, el trabajo en equipo, el respeto del medio ambiente y su conservación, generando actitudes sobre la ciencia como su postura crítica, la reflexión sobre el trabajo científico y actitudes como la socialización de resultados, etc.

Para Gavidia (2008), la escuela tiene un papel socializante y su función es formar personas críticas con capacidad para tomar decisiones y hacer frente a los problemas cotidianos de la sociedad actual. Estudios como el de Fernández, Rodríguez y Casal (1999) han mostrado la efectividad del trabajo de campo no solo en la comprensión de conceptos ecológicos, sino por su incidencia en el desarrollo de actitudes más favorables en la defensa de los ecosistemas. Se trata de sobrepasar las Salidas de Campo tradicionales en las que predomina el aprendizaje conceptual (LAVIE ALON; TAL, 2017) y favorecer, a partir del acercamiento real de los objetos de estudio, su conservación que puede facilitarse con otras actividades de campo como la fotografía, los videos y la colecta de material desechado por los organismos (GRILLI-SILVA, 2018).

El hecho de compartir con el alumnado durante un mes el contacto con la naturaleza y con la realidad educativa de la ciudad de Neiva, permitió a los futuros docentes identificar las dificultades en el aprendizaje de comportamientos, actitudes y valoraciones que poseen los alumnos de educación secundaria. Desde la perspectiva del CPP, varios autores manifiestan que en la experiencia docente y en la práctica educativa se configura el saber profesional de los maestros (PORLÁN; RIVERO, 1998; PORLÁN et al., 2001).

\section{Categoría: Planificación}

[Eres un profesor titular de una Institución Educativa de la ciudad de Neiva, te encuentras enseñando Biología para el grado noveno y tienes en mente realizar una Salida de Campo. Al respecto, ¿Qué tendrías en cuenta para realizarla?] Respuestas Pretest:

"Tendría en cuenta la metodología a seguir; el tema en específico, recursos económicos, que el lugar a visitar sea apto para el objetivo de la práctica, lo materiales para realizarla..."

Veinte y tres $(85,2 \%)$ docentes en formación hacen afirmaciones semejantes a la expuesta Asuntos como la Guía de campo (2 estudiantes), las Ideas previas (6 estudiantes) y la Motivación del alumnado (5 estudiantes) son escasamente tenidas en cuenta por el profesorado en formación. Considerar que para planificar la Práctica de Campo 
es suficiente con aspectos de tipo logístico, podría relacionarse con enfoques didácticos de tipo tradicional, en los cuales la actividad se centra en el profesor, con un grado de participación del alumnado mínimo. Esta concepción sigue siendo la más frecuente (PEDRINACl, 2012).

Respuestas Postest que atienden a las Ideas Previas:

"Estado del conocimiento del estudiante, aptitudes y destrezas, relevancia del tema, motivación hacia los estudiantes, debilidades y fortalezas tanto del estudiante como del docente..."

Con relación al inicio del proceso formativo donde tan solo $6(22,2 \%)$ de los futuros docentes manifestaban que tendrían en cuenta las Ideas previas de su alumnado, $13(48,2 \%)$ de ellos consideran este aspecto en la fase posterior de nuestra intervención didáctica. La comparación entre el pre y post test arrojó un p-valor $=0.05$.

Para Behrendt y Franklin (2014), el profesorado debe tener en cuenta las ideas previas de sus alumnos y permitir un alto grado de su participación en la actividad de campo, de forma que genere en ellos mayor motivación. Para Del Carmen y Pedrinaci (1997) y Dourado y Leite (2013), es ideal que las actividades de campo sean planificadas por el profesor desde una perspectiva de tipo constructivista, con un enfoque por descubrimiento. Estudios como el de Martín del Pozo, Rivero y Azcárate (2014) han puesto de manifiesto la importancia de abordar el manejo de las ideas previas y su relevancia didáctica, en los programas de formación inicial del profesorado de ciencias naturales.

Respuestas Postest que atiende a las Motivaciones:

"Tendría en cuenta el tema a desarrollar para así proponer estrategias didácticas las cuales me faciliten tener un buen manejo del curso y los estudiantes se motiven ante éste..."

A diferencia de los cinco docentes en formación (18,5\%) que habían señalado este aspecto al inicio del proceso formativo, 15 (55,5\%) pasaron a contemplarla después de la intervención didáctica. La comparación entre el pre y post test arrojó un p-valor = 0.009 , mostrando así un alto grado de significatividad. Para Hurley (2006) y Dillon et al. (2006), el alumnado puede generar expectativas sobre las actividades que realizarán en el campo y así aumentar su motivación hacia el trabajo. Afirman que una Práctica de Campo adecuadamente concebida ofrece al alumnado la posibilidad de desarrollar valoraciones sobre su propio aprendizaje. El acercamiento de los futuros docentes a la realidad educativa fue fundamental para que conocieran de primera mano que la motivación es uno de los aspectos fundamentales en el aprendizaje de las ciencias naturales.

\section{Categoría: Aporte a la Formación Docente}

[¿Qué aportan las Prácticas de Campo a tu formación como docente de Ciencias Naturales?]

Respuestas Pretest:

"Me ayudan a afianzar mis conocimientos en ciencias naturales...". 
De manera mayoritaria, 16 futuros docentes (59,2\%) conciben que el principal aporte de las Prácticas de Campo en su formación como profesores ha sido que han aprendido Biología. Ideas minoritarias son la Experiencia docente (2 estudiantes-7,4\%) y la Enseñanza de la Biología (7 estudiantes-26\%). Generalmente el profesorado en su formación inicial ha participado como alumnado en las Salidas de Campo, sin tener experiencias en la planificación y complejidad de enseñar fuera del aula (TAL; MORAG, 2009). Lavie Alon y Tal (2016; 2017) afirman que son escasas las investigaciones sobre cómo el profesorado emplea el medio natural en la enseñanza.

Respuestas Postest que atienden a la Enseñanza de la Biología:

"Aprovechar los espacios educativos fuera del aula de clase para hacer ciencias..." "la socialización con los estudiantes fuera de la clase me permitiría conocerlos mejor..."

Los profesores en formación ( 21 casos $-77,7 \%$ ) destacan el papel fundamental de las Prácticas de Campo como una estrategia de enseñanza de gran importancia que esperan desarrollar de manera activa en su ejercicio docente. La comparación entre el pre y post test arrojó un p-valor $=0.003$ mostrando así un alto grado de significatividad entre los datos. El profesorado en formación destaca el impacto y la aportación de esta actividad en el aprendizaje de su alumnado facilitando la integración de aprendizajes conceptuales, procedimentales y actitudinales, que les permitirá conocer mejor las características de su alumnado. Ello corresponde con los planteamientos de Dourado (2006) y Del Carmen (2011) que conciben la Práctica de Campo como una estrategia de enseñanza fundamental de la Biología por lo que el futuro profesorado debe ser consciente que las Prácticas de Campo son una estrategia de enseñanza fundamental y contribuyen en la construcción del CPP (COSTILLO et al., 2014; BEHRENDT; FRANKLIN, 2014).

Respuesta Postest que atiende al Desarrollo profesional:

"Una gran satisfacción por haber llevado a cabo una actividad que puede llegar a marcar la vida de los estudiantes..." "Inicialmente experiencia al aplicar una forma diferente de enseñanza, también una estructuración del modelo de enseñanza y método de evaluación..."

Aquí 15 (55,5\%) docentes en formación explicitan que el diseño, puesta en marcha y evaluación de una Práctica de Campo con estudiantes de secundaria había tenido incidencia en su desarrollo profesional. La comparación entre el pre y post test arrojó un $p$-valor $=0.029$, lo que indica diferencia significativa entre los resultados del pre y del post test. Los asistentes al Seminario identificaron que las Prácticas de Campo, como estrategia de enseñanza de la Biología, les podría favorecer el desarrollo de los lazos afectivos con sus alumnos, la satisfacción profesional por el adecuado manejo de las actividades de aprendizaje, la ética y la imagen de docente integral y su aportación a la institución educativa donde llevaron a cabo la actividad. La planificación, desarrollo y evaluación de las Prácticas de Campo en la enseñanza de la Biología permite al docente en formación generar actitudes de auto-eficacia, confianza en su quehacer como docente (ATESKAN; LANE, 2016) y desarrollo profesional (TAL, 2001). En palabras de Fernán- 
dez, Costillo y Amórtegui (2015), se trata de que los futuros maestros pongan en evidencia sus concepciones, pero a través de discusiones puedan detectarlas, modificarlas y replantearlas.

\section{CONCLUSIONES}

Pese a que el profesorado en formación suele participar en Prácticas de Campo durante su formación inicial, sus concepciones acerca de las implicaciones didácticas de esta estrategia de enseñanza de la Biología son reduccionistas, pues entienden que es suficiente con que el profesor seleccione un lugar y una temática para llevar a cabo una Práctica de Campo, en donde principalmente lo que hacen los alumnos es corroborar lo que el profesor ha transmitido previamente en el aula. Consideramos que una propuesta formativa para el profesorado que contemple el diseño, desarrollo y evaluación de una Práctica de Campo para la enseñanza de una temática biológica dirigida al alumnado de educación secundaria, permite la configuración de un CDC específico. En el desarrollo del seminario se hicieron explicitas sus concepciones e intenciones con relación al currículo, los propósitos de la enseñanza, la evaluación del aprendizaje, los saberes de los alumnos, etc., centrado todo ello en las Prácticas de Campo, lo cual es considerado por Park y Chen (2012) y Gess-Newsome (2015) como elementos del CDC fundamentales para la construcción del CPP. Las Prácticas de Campo facilitan que el alumnado aprenda entrando en contacto directo con los fenómenos biológicos, no solo a través de la mera observación sino mediante el análisis directo, el desarrollo de habilidades y destrezas científicas y la generación de actitudes y comportamientos en pro de la conservación de la diversidad biológica, y de los ecosistemas.

Por último, destacamos que, en términos del desarrollo profesional, es necesario que la formación inicial del profesorado incluya las Prácticas de Campo, y entienda la necesidad de su planificación, la participación en ellas, así como la evaluación en su currículo de aprendizaje (BEHRENDT; FRANKLIN, 2014). A manera de proyección, consideramos importante continuar investigando acerca de las actividades de campo que realiza el profesorado de ciencias al enseñar biología en las escuelas, más desde la caracterización de sus prácticas docentes que desde sus concepciones. Por otra parte, la generación de pensamiento crítico, el desarrollo de la argumentación, los procesos de modelización, etc. siguen siendo un amplio campo de estudio a conseguir a través de las Prácticas de Campo.

\section{REFERENCIAS}

ÁLVAREZ, J.; JURGENSON, G. Cómo hacer investigación cualitativa. Fundamentos y metodología. México Distrito Federal: Paidós Educador, 2003.

AMÓRTEGUI, E. Concepciones sobre prácticas de campo y su relación con el conocimiento profesional del profesor, de futuros docentes de biología de la Universidad Pedagógica Nacional. 2011. Tesis (Maestría en Educación) - Facultad de Educación, Universidad Pedagógica Nacional, Bogotá, Colombia, 2011.

AMÓRTEGUI, E.; CORREA, M. Las Prácticas de Campo Planificadas en el Proyecto Curricular de Licenciatura en Biología de la Universidad Pedagógica Nacional. Caracterización desde la perspectiva del Conocimiento Profesional del Profesor de Biología. 1. ed. Bogotá: Editorial Fundación Francisca Radke, 2012. 
AMÓRTEGUI, E.; MAYORAL, O.; GAVIDIA, V. Aportaciones de las Prácticas de Campo en la formación del profesorado de Biología: un problema de investigación y una revisión documental. Didáctica de las Ciencias Experimentales y Sociales, n. 32, p. 153-169, 2017. DOI: https://doi.org/10.7203/dces.32.9940

ARTOLA, E. C.; MAYORAL, L. E.; BENARROCH, A. Dificultades de aprendizaje de las representaciones gráficas cartesianas asociadas a biología de poblaciones en estudiantes de educación secundaria. Un estudio semiótico. Revista Eureka sobre Enseñanza y Divulgación de las Ciencias, n. 13, p. 36-52, 2016.

ATEŞKAN, A.; LANE, J. Promoting field trip confidence: teachers providing insights for pre-service education, European Journal of Teacher Education, n. 39, p. 190-201, 2016. DOI: https://doi.org/10.1080/0261 9768.2015.1113252

BANET, E. La enseñanza y el aprendizaje del conocimiento biológico. In: PERALES, J.; CAÑAL, P. (coord.). Didáctica de las ciencias experimentales. Teoría y práctica de la enseñanza de las ciencias. Alcoy: Marfil, 2000. p. 449-478.

BEHRENDT, M.; FRANKLIN, T. A review of research on school field trips and their value in education. International Journal of Environmental and Science Education, n. 9(3), p. 235-245, 2014. DOI: https:// doi:10.12973/ijese.2014.213

BRODY, M. Learning in nature. Environmental Education Research, n. 11(5), p. 603-621, 2005. DOI: https:// doi.org/10.1080/13504620500169809

COHEN, L.; MANION, L.; MORRISON, K. Research methods in education. New York: Routledge, 2011.

COSTILLO, E. et al. Utilización de la modelización para trabajar las salidas al medio natural en profesores en formación de educación secundaria. Bio-grafía: Escritos Sobre la Biología y su Enseñanza, n. 7(13), p. 165-175, 2014. DOI: http://dx.doi.org/10.17227/20271034.vol.7num.13bio-grafia165.175

DEL CARMEN, L.; PEDRINACI E. El uso del entorno y el trabajo de campo. In: DEL CARMEN, L. (coord.). La enseñanza y el aprendizaje de las ciencias de la naturaleza en la educación secundaria. Barcelona: Editorial Horsori, 1997. p. 133-154.

DEL CARMEN, L.. El lugar de los trabajos prácticos en la construcción del conocimiento científico en la enseñanza de la Biología y la Geología. In: CAÑAL, P. (coord.). Didáctica de la Biología y la Geología. Barcelona. Graó, 2011. p. 91-108.

DEL TORO, R.; MORCILLO, J. Las actividades de campo en educación secundaria. Un estudio comparativo entre Dinamarca y España. Enseñanza de las Ciencias de la Tierra, n. 19(1), p. 39-47, 2011.

DILLON, J. et al. The value of outdoor learning: Evidence from research in the UK and elsewhere. School Science Review, n. 87, p. 107-111, 2006.

DOURADO, L. Concepções e práticas dos professores de ciências naturais relativas à implementação integrada do trabalho laboratorial e do trabalho de campo. Revista Electrónica de Enseñanza de las Ciencias, n. 5(1), p. 192-212, 2006.

DOURADO, L.; LEITE, L. Field activities, science education and problem-solving. Procedia Social and Behavioral Sciences, n. 106(10), p. 1.232-1.241, 2013. DOI: https://doi.org/10.1016/j.sbspro.2013.12.138

FERNÁNDEZ, R.; RODRÍGUEZ, L.; CASAL, M. Relationship between Ecology Fieldwork and Student Attitudes toward Environmental Protection. Journal of Research in Science Teaching, n. 36(4), p. 431-453, 1999.

FERNÁNDEZ, N.; COSTILLO, E.; AMÓRTEGUI, E. Concepciones sobre trabajos prácticos de campo en la enseñanza de la Biología de futuros docentes de Colombia, España y Argentina. Biografía. Escritos sobre la Biología y su Enseñanza, Número extraordinario. ENCUENTRO NACIONAL DE EXPERIENCIAS EN ENSEÑANZA DE LA BIOLOGÍA Y LA EDUCACIÓN AMBIENTAL, 8., CONGRESO NACIONAL DE INVESTIGACIÓN EN ENSEÑANZA DE LA BIOLOGÍA, 3., 2015, p. 1.019-1.032. DOI: http://dx.doi.org/10.17227/20271034. vol.Onum.Obio-grafia1019.1032

GAVIDIA, V. Las actitudes en la educación científica. Didáctica de las Ciencias Experimentales y Sociales, $\mathrm{n}$. 22, p. 53-66, 2008. DOI: https://doi.org/10.7203/dces..2420

GESS-NEWSOME, J. A model of teacher professional knowledge and skill including PCK: Results of the thinking from the PCK Summit. In: BERRY, A.; FRIEDRICHESEN, P.; LOUGHRAN, J. Re-examining Pedagogical Content Knowledge in Science Education. New York: Routledge, 2015. p. 28-42.

GLACKIN, M. "Risky Fun” or "Authentic Science”? How Teachers' Beliefs Influence their Practice during a Professional Development Programme on Outdoor Learning. International Journal of Science Education, n. 38(3), p. 409-433, 2016. DOI: https://doi.org/10.1080/09500693.2016.1145368

GRILLI-SILVA, J. El material natural en la Biología escolar. Consideraciones éticas y didáctica sobre las actividades prácticas de laboratorio. Revista Eureka sobre Enseñanza y Divulgación de las Ciencias. n. 15(1), p. 1.104, 2018. DOI: 10.25267/Rev_Eureka_ensen_divulg_cienc.2018.v15.i1.1104 
HAMILTON-EKEKE, J. Relative effectiveness of expository and field trip methods of teaching on students' achievement in ecology. International Journal of Science Education, n. 29(15), p. 1.869-1.889, 2007. DOI: https://doi.org/10.1080/09500690601101664

HURLEY, M. Field Trips as Cognitive Motivators for High Level Science Learning. The American Biology Teacher, 68(6), p. 61-66, 2006. DOI: https://doi.org/10.1662/0002-7685(2006)68[61:FTACMF]2.0.CO,2

JIMÉNEZ, M. La enseñanza y el aprendizaje de la biología. In: JIMÉNEZ, M. (coord.). Enseñar Ciencias. Barcelona: Grao, 2003. p. 119-146.

LAVIE ALON, N.; TAL, T. Student Self-Reported Learning Outcomes of Field Trips: The pedagogical impact. International Journal of Science Education, n. 37(8), p. 1.279-1.298, 2015. DOI: https://doi.org/10.1080/0 9500693.2015.1034797

LAVIE ALON, N.; TAL, T. Teachers as Secondary Players: Involvement in Field Trips to Natural Environments. Research in Science Education, 47(4), p. 869-877, 2016. DOI: 10.1007/s11165-016-9531-0

LAVIE ALON, N.; TAL, T. Field trips to natural environments: how outdoor educators use the physical environment. International Journal of Science Education, part B. n. 7(3), p. 237-252, 2017. DOI: 10.1080/21548455.2016.1250291

MAGNTORN, O.; HELLDÉN, G. Students' ability to read nature. Reflections on their own learning in ecology. International Journal of Science Education, 27(10), p. 1.229-1.254, 2005. DOI: https://doi. org/10.1080/09500690500102706

MAGNTORN, O.; HELLDÉN, G. Reading New Environments: Students' ability to generalize their understanding between different ecosystems, International Journal of Science Education, 29(1), p. 67-100, 2007. https://doi.org/10.1080/09500690600708543

MARTÍN DEL POZO, R.; RIVERO, A.; AZCÁRATE, M. Las concepciones de los futuros maestros sobre la naturaleza, cambio y utilización didáctica de las ideas de los alumnos. Revista Eureka Sobre Enseñanza y Divulgación de las Ciencias, n. 11(3), p. 348-363, 2014. DOI: http://hdl.handle.net/10498/16588

MCLAUGHLIN, J.; JOHNSON, D. Assessing the Field Course Experiential Learning Model: Transforming Collegiate Short-term Study Abroad Experiences into Rich Learning Environments. The Interdisciplinary Journal of Study Abroad. XVIII. p. 65-85, 2006.

MORAG, O.; TAL, T. Assessing Learning in the Outdoors with the Field Trip in Natural Environments (FiNE) Framework. International Journal of Science Education, n. 34(5), p. 745-777, 2012. DOI: https://doi.org/1 0.1080/09500693.2011.599046

MORAG, O.; TAL, T.; ROTEM-KEREN, T. Long-Term Educational Programs in Nature Parks: Characteristics, Outcomes and Challenges. International Journal of Environmental and Science Education, n. 8(3), p. 427449, 2013. DOI: 10.12973/ijese.2013.213a

PARK, S.; CHEN, Y. Mapping Out the Integration of the Components of Pedagogical Content Knowledge (PCK): Examples From High School Biology Classrooms. Journal of Research in Science Teaching, n. 49(7), p. 922-941, 2012. DOI 10.1002/tea.21022.

PEDRINACI, E. Trabajo de campo y aprendizaje de las ciencias. Alambique Didáctica de las Ciencias Experimentales, n. 71, p. 81-89, 2012.

PÉREZ, G. Investigación cualitativa. Retos e interrogantes (II técnicas y análisis de datos). Madrid: La Muralla, 1994

PORLÁN, R.; RIVERO, A. El conocimiento de los profesores: una propuesta formativa en el área de ciencias. Sevilla: Díada, 1998.

PORLÁN, R. et al. La relación teoría-práctica en la formación permanente del profesorado. Sevilla: Díada Editora, 2001.

PUENTES, M. Propuesta de un sistema de categorías para el estudio del Trabajo Práctico en la enseñanza de la Biología. 2008. Tesis (Especialización en Enseñanza de la Biología) - Universidad Pedagógica Nacional, Facultad de Educación, Bogotá, Colombia, 2008.

RIVERO, A. et al. Cambio del conocimiento sobre la enseñanza de las ciencias de futuros maestros. Enseñanza de las Ciencias, n. 35(1), p. 29-52, 2017. DOI: https://doi.org/10.5565/rev/ensciencias.2068

TAL, T. Incorporating field trips as science learning environment enrichment, an interpretive study. Learning Environment Research, 4(1), p. 25-49, 2001.

TAL, T.; MORAG, O. Reflective Practice as a Means for Preparing to Teach Outdoors in an Ecological Garden. Journal of Science Teacher Education, 20(3), p. 245-262, 2009. DOI 10.1007/s10972-009-9131-1.

VAN DRIEL, J.; BERRY, A.; MEIRINK, J.. Research on Science teacher knowledge. In: LEDERMAN (Ed.). Handbook of Research on Science Education. 2nd ed. London: Taylor \& Francis, 2014. p. 848-870. 


\section{ANEXOS}

\section{Anexo 1 - Programa del seminario Las Prácticas de Campo en la enseñanza de la biología y la formación docente}

\begin{tabular}{|c|c|c|}
\hline SESIÓN & ACTIVIDAD & OBJETIVO \\
\hline $\begin{array}{l}\text { 1. ¿QUÉ ES ESO DE } \\
\text { SALIR AL CAMPO? }\end{array}$ & $\begin{array}{l}\text { 1. ¿Qué pienso del trabajo } \\
\text { de campo? } \\
\text { 2. ¿Cómo representamos } \\
\text { nuestras ideas sobre las } \\
\text { Prácticas de Campo? } \\
\text { 3. ¿Qué importancia tiene } \\
\text { el trabajo de campo para } \\
\text { un país como Colombia? }\end{array}$ & $\begin{array}{l}\text { Explicitar las concepciones iniciales del futuro profesorado } \\
\text { sobre diversos aspectos de las Prácticas de Campo. La } \\
\text { actividad se desarrolla primero de manera individual, } \\
\text { posteriormente en pequeños grupos y finalmente se realiza } \\
\text { una socialización. Se emplean formularios y la realización de } \\
\text { dibujos. Para la Actividad 3, se analizan segmentos de videos } \\
\text { sobre Charles Darwin y sobre Francisco José de Caldas en la } \\
\text { Expedición Botánica. }\end{array}$ \\
\hline $\begin{array}{l}\text { 2. ¿QUÉ OCURRE } \\
\text { CON LAS “PRÁCTICAS } \\
\text { EXTRAMUROS”? }\end{array}$ & $\begin{array}{l}\text { 4. ¿Qué tipo de actividades } \\
\text { son las "Prácticas } \\
\text { Extramuros"? } \\
\text { 5. Primera entrega del } \\
\text { diseño de la Práctica de } \\
\text { Campo }\end{array}$ & $\begin{array}{l}\text { Se analizan varias actividades de educación fuera de la } \\
\text { escuela (Centro de ciencia interactiva, museo de historia } \\
\text { natural y granja); luego se analizan las Salidas de Campo que } \\
\text { los futuros profesores han realizado durante sus primeros } \\
\text { años de formación docente, específicamente las relacionadas } \\
\text { con Biología (sistemática, botánica, zoología, ecología). Los } \\
\text { futuros docentes realizan su primera propuesta de Práctica de } \\
\text { Campo. }\end{array}$ \\
\hline EXTRAORDINARIA & $\begin{array}{l}\text { Práctica Extramuro a la } \\
\text { ciudad de Medellín. Se } \\
\text { visitan Jardín Botánico, } \\
\text { Centro de ciencia } \\
\text { interactivo, Zoológico y } \\
\text { Universidad de Antioquia. }\end{array}$ & $\begin{array}{l}\text { Identificar diversos escenarios de enseñanza-aprendizaje } \\
\text { de las ciencias naturales y reflexionar sobre sus fortalezas y } \\
\text { debilidades en el contexto de su formación docente. }\end{array}$ \\
\hline $\begin{array}{l}\text { 3. ¿QUÉ TENEMOS } \\
\text { EN CUENTA EN UNA } \\
\text { SALIDA DE CAMPO? }\end{array}$ & $\begin{array}{l}\text { 6. ¿Qué criterios podemos } \\
\text { tener en cuenta para el } \\
\text { diseño de una Práctica de } \\
\text { Campo? }\end{array}$ & $\begin{array}{l}\text { Los profesores-estudiantes analizan guías de salidas de campo } \\
\text { diseñadas y aplicadas por profesores de su misma licenciatura. } \\
\text { Posteriormente se introduce nuestra propuesta sobre los } \\
\text { aspectos que consideramos pueden orientar el diseño de } \\
\text { Prácticas de Campo y se analizan de nuevo. }\end{array}$ \\
\hline $\begin{array}{l}\text { 4. ALGUNAS } \\
\text { INVESTIGACIONES } \\
\text { SOBRE SALIDAS DE } \\
\text { CAMPO EN EL HUILA }\end{array}$ & $\begin{array}{l}\text { 7. Conociendo algunas } \\
\text { investigaciones } \\
\text { 8. Segunda entrega del } \\
\text { diseño de la Práctica de } \\
\text { Campo }\end{array}$ & $\begin{array}{l}\text { Se trata de reconocer desde la experiencia de los graduados } \\
\text { de la licenciatura que se desempeñan ahora como profesores } \\
\text { de ciencias naturales en instituciones educativas del Huila, su } \\
\text { experiencia con relación al desarrollo de Prácticas de Campo. } \\
\text { Realizan la segunda entrega de su diseño de la Salida de } \\
\text { Campo; y a diferencia de la anterior, se centran en aspectos } \\
\text { didácticos que han sido presentados y socializados en el curso. }\end{array}$ \\
\hline $\begin{array}{l}\text { 5. ¿CÓMO VAMOS } \\
\text { PREPARANDO EL } \\
\text { TRABAJO? }\end{array}$ & 9. Asesoría & $\begin{array}{l}\text { El profesor guía del seminario realiza un acompañamiento y } \\
\text { asesoría a las propuestas de campo diseñadas por los futuros } \\
\text { docentes: la planificación, el planteamiento de la guía de } \\
\text { campo, las actividades previas y posteriores en el salón de } \\
\text { clases, etc. }\end{array}$ \\
\hline $\begin{array}{l}\text { 6. ¿CÓMO FUE LA } \\
\text { SALIDA DE CAMPO? }\end{array}$ & $\begin{array}{l}\text { 10. Observando el trabajo } \\
\text { de mis compañeros } \\
\text { 11. ¿Cómo evalúo mi } \\
\text { trabajo? } \\
\text { 12. Tercera entrega: } \\
\text { Informe final }\end{array}$ & $\begin{array}{l}\text { Esta sesión, se lleva a cabo luego de que todos los grupos } \\
\text { han realizado sus Prácticas de Campo con los alumnos de } \\
\text { secundaria y han evaluado su aprendizaje. Se trata de realizar } \\
\text { una reflexión sobre su puesta en marcha, sus fortalezas, } \\
\text { valoraciones, debilidades y aspectos por mejorar. Se propone, } \\
\text { una coevaluación, una heteroevaluación y una autoevaluación } \\
\text { (a través de la observación de diversos segmentos de videos } \\
\text { que muestra el desarrollo de sus Salidas de). Se trata de } \\
\text { culminar la experiencia formativa, centrándose en la reflexión } \\
\text { sobre su propio desempeño. }\end{array}$ \\
\hline
\end{tabular}

Fuente: Elaborado por los autores. 